\title{
ANÁLISIS DEL EMPLEO DE DOCUMENTALES EN EL ÁREA DE ORGANIZACIÓN DE EMPRESAS: Un instrumento para acercar a los alumnos a la realidad empresarial
}

Rocío González Sánchez

Miguel Blanco Callejo

Profesores de Organización de Empresas

Universidad Rey Juan Carlos

\begin{abstract}
Resumen
En un entorno educativo en el que cada vez se dispone de más recursos y medios de carácter audiovisual, el presente trabajo pretende mostrar la aplicación docente de películas documentales como mecanismo de transmisión de conocimientos y la mejora de los niveles de aprendizaje conseguidos por los estudiantes. El trabajo expone en primer lugar la justificación del empleo de esta técnica en un contexto universitario y describe una experiencia práctica desarrollada por dos profesores de la Universidad Rey Juan Carlos enfocada a la docencia en materias del área de Organización y Dirección de Empresas. El resultado obtenido permite afirmar que la utilización de documentales en la formación sobre organización empresarial ha permitido acercar al alumno una visión completa del funcionamiento de una empresa: estrategia, RRHH, marketing, producción, finanzas y gobierno corporativo y conseguir en el mismo mayores niveles de motivación y satisfacción que otras técnicas docentes tradicionales.
\end{abstract}

\section{Palabras clave}

Documental - video - estudio de casos - material multimedia - Organización de Empresas aprendizaje 


\section{Abstract}

Current educational environment is defined by an increasing trend in the use of audiovisual resources and media. The aim of this piece of work is to show in a practical form the use of documentary films as a valid tool oriented to knowledge transmission and improvement of student's learning levels. Firstly, the paper justifies the use of this educational tool in a college context and secondly describes a practical experience of two business management professors in Rey Juan Carlos University. The results of the study confirm that the use of documentary films in teaching management have allowed students to get closer to an integral view of the companies: strategy, human resources management, marketing, operation management, finance and corporate governance and to obtain greater levels of motivation and satisfaction than other traditional educational tools.

\section{Key words}

Documentary film - video - case study - multimedia resources - Business management - learning

\section{Introducción Teórica: el empleo de vídeos documentales para la docencia}

Uno de los problemas más importantes con los que se encuentran los alumnos en las asignaturas con un carácter introductorio en la enseñanza sobre organización de empresas dentro del ámbito universitario es la incapacidad de alcanzar una perspectiva global de la empresa. Esto es debido, en gran medida, a la existencia de un elevado número de temas sobre áreas muy diversas de las organizaciones y que en ocasiones pueden parecer escasamente integrados. Además, la fórmula habitual a través de la cuál se imparten estos contenidos suelen apoyarse en la técnica docente de la “lección magistral”. Esta circunstancia hace que, que los alumnos mantengan un escaso contacto directo con las empresas, que se limita principalmente a aplicaciones prácticas derivadas de estudios de casos y de noticias de prensa, que emplean los profesores para apoyar las explicaciones teóricas (Bonache, 1999). 
En buena medida, la literatura sostiene que a través de la introducción de nuevas herramientas y aplicaciones se pueden obtener instrumentos que sirvan de apoyo a la enseñanza (Aguilera y Méndiz, 2003; Cannings y Talley, 2003). Sin embargo, numerosos estudios, y en particular el nuevo Espacio Europeo de Educación Superior, propugnan un enfoque más práctico y activo de docencia y destacan la notable preocupación de la comunidad educativa por las limitaciones a las que se enfrentan los profesores a la hora de integrar las diversas metodologías en los programas de enseñanza tradicional (Perry y Talley, 2001; Sprague et al., 1998). Este trabajo pretende, a través de una aplicación práctica que muestra el empleo de una novedosa metodología de apoyo a la enseñanza universitaria dentro del área de Organización de Empresas, ofrecer nuevas alternativas a los docentes universitarios y facilitar e incentivar la realización de este tipo de actividades por parte de profesores de esta área y otras áreas dentro de la Universidad. El trabajo defiende que con el empleo de esta técnica docente se podría alcanzar una mejor convivencia e integración de diversas tecnologías/metodologías, mejorando y dinamizando el desarrollo de las clases teóricas y consiguiendo mayores niveles de motivación de los alumnos.

El empleo de películas de diversos formatos y temáticas como una modalidad de material audiovisual docente, que permite mejorar la formación y el aprendizaje de los estudiantesespectadores de cualquier nivel educativo, ha sido analizado por diversos trabajos en la literatura reciente (Dong y Li, 2005; Earley, 1987; Götz, 2005; Philips, 2002). Uno de los aspectos que destacan estas investigaciones es la eficacia y eficiencia de esta técnica como un medio que consigue a través del aprendizaje experimental y la motivación que despierta en los estudiantes elevar la transmisión de conocimientos a los alumnos. La investigación en este ámbito ha sido tan importante, que se puede hablar de toda una corriente de investigación en la literatura que se remonta a las primeras aplicaciones de esta herramienta ${ }^{1}$ (Grierson, 1936; Kolb y Fry, 1975; Mercey, 1939; Moffat y Rich, 1951; Moss, 1979; Poltorak, 1992; Raack, 1972).

En particular este trabajo se va a centrar en un tipo específico de material audiovisual, la utilización de “films” de carácter documental (Philips, 2002). El alcance del trabajo se limita al

\footnotetext{
${ }^{1}$ Grierson en 1936 ofrecía una visión entusiasta sobre el empleo de películas dentro de las aulas: “con las películas se puede disponer de un instrumentos mucho más adecuado a los propósitos específicos de la educación que cualquier otro ámbito artístico” (Rack, 1972:109).
} 
estudio de vídeos de carácter documental que describen casos concretos de empresas y organizaciones que pueden ser empleados para la didáctica a nivel universitario y, concretamente, dentro del campo de Organización y Dirección de Empresas. En este sentido, las películas de carácter documental pueden desempeñar un papel muy importante para enriquecer la experiencia formativa de los estudiantes (Dong y Li, 2005; Raack, 1973). Esto se debe a que la combinación de sonido e imágenes en movimiento, describiendo cualquier situación, capta la atención de cualquier espectador y más en particular de un estudiante en un entorno educativo (Rack, 1972). A pesar de esta circunstancia, el acceso y el empleo de videos de carácter documental ha estado tradicionalmente más limitado debido a la mayor dificultad de conseguir dichos recursos en comparación con textos u otro tipo de material bibliográfico. Sin embargo, Moss (1979) ya detectaba a principios de la década de los ochenta que cada vez era más frecuente que las bibliotecas de las universidades y las aulas dispusieran de recursos audiovisuales así como de equipos que permitían la reproducción de los mismos dentro de las aulas, lo que promovía su utilización en el desarrollo de las clases. Otros estudios se apoyan en estos medios, disponibles en muchas universidades, para realizar proyectos de investigación que mejoran el desarrollo de cursos de formación, por ejemplo a través de la formación mediante técnicas de televisión educativa y cursos on-line con acceso de vídeos bajo demanda a través de Internet (Heeler y Hardy, 2005).

\subsection{Delimitación de conceptos}

Un film documental, en palabras de Bill Nichols representa una forma de “discurso de sobriedad”. Este tipo de materiales que pueden incluir contenidos relacionados con diversos ámbitos científicos son “discursos” que pretenden describir lo real para contar la verdad (Goldmillow y Shapiro, 1997). Wordnet describe a los documentales como películas o programas de televisión que presentan una serie de hechos sobre una persona o un determinado suceso. Marqués (1999) define los vídeos educativos de carácter documental como vídeos que presentan ordenadamente información sobre un tema concreto. En cualquier caso, se puede afirmar que los documentales abarcan un amplio espectro de temas que incluyen ciencia, historia, economía, sucesos políticos y sociales y muchos otros aspectos y actualmente se han convertido en uno de los más importantes materiales de aprendizaje multimedia (Dong y Li, 2005) e incluso han sido empleados como un nuevo medio con gran potencial para el entrenamiento de empleados (Smith et al., 1999). Sin 
embargo, debido a su carácter en ocasiones escasamente estructurado y a sus especiales características representan un material cuyo acceso es mucho más difícil que los documentos basado en texto y su aplicación efectiva ha sido limitada en el ámbito educativo (Dong y Li, 2005).

Tomando como punto de partida las definiciones presentadas y los trabajos de investigación que han analizado el empleo docente de esta herramienta, un aspecto relevante del vídeo documental es que pretende conseguir mayores niveles de aprendizaje de los individuos sobre el tema presentado. Partiendo de la dificultad de definición de este término, se propone la siguiente definición de aprendizaje "la adquisición relativamente perdurable de conocimientos, capacidades, habilidades, actitudes y modelos de comportamiento o su cambio debido a la experiencia” (Brockhaus, 1998:310). Si el objetivo de un programa académico/docente es la transmisión de una serie de conocimientos establecidos, entonces el éxito de dicho esfuerzo puede ser determinado precisamente a través del empleo de herramientas de pre-test y de post-test. Tal aproximación se basa, de forma implícita, en un modelo de recepción a través un soporte audiovisual en la cual ciertos contenidos, o partes de los contenidos, son transmitidos por el programa y recibidos por los estudiantes y se debe verificar el efecto que tiene éste sobre los mismos (Gotz, 2005).

Cabe destacar, en cualquier caso, una limitación relacionada con el empleo de esta herramienta que ha sido detectada en los estudios que han relacionado el empleo de soportes y materiales audiovisuales enfocados a la docencia y los diferentes niveles de aprendizaje. Estos trabajos han demostrado que el aprendizaje a través de estos medios no se puede generalizar a toda una población de estudiantes, ya que dado el carácter personal y particular del aprendizaje representa una experiencia individual por parte de cada uno de los estudiantes y debe ser controlada individualizadamente (Bachmair, 1996; Gotz, 2005; Mikos, 2001).

\subsection{Análisis del empleo docente de material audiovisual}

La aportación pedagógica del empleo de material audiovisual de carácter documental ha sido un aspecto ampliamente reconocido en la literatura (Mercey, 1939; Moss, 1979; Raack, 1972; Rogers y Scaife, 1997; Smith et al, 1999). Así, ya en un estudio de finales de los años 30, Mercey (1939) reconocía las virtudes de emplear material audiovisual documental frente a textos escritos 
como un medio que podía estimular y motivar, en mayor medida, a los estudiantes y, de este modo, conseguir transmitirles aspectos que serían mucho más difíciles empleando otro tipo de materiales. Posteriormente, Moss (1979: 1013), centrándose en el ámbito económico, reconocía que "los medios audiovisuales documentales habían demostrado su potencial como un medio de transmisión de información científica sobre aspectos de carácter económico de un modo muy interesante y efectivo”. En esta línea, Raack (1972) afirmaba que se podían alcanzar importantes "beneficios educativos” a través del uso de documentales en las aulas. Los trabajos destacan que desde el primer momento se puede observar un mayor interés de los usuarios si el material tiene carácter audiovisual/multimedia, $\mathrm{y}$, de hecho, los alumnos en la práctica totalidad de los casos prefieren el empleo de material audiovisual a la presentación de la actividad en texto (Rogers y Scaife, 1997).

Otra importante corriente investigadora analiza los factores considerados importantes en el desarrollo de habilidades del profesor para controlar las interacciones en clase, considerando el material audiovisual proyectado como una herramienta efectiva (Gamoran y Van Es, 2002; Putnam y Borko, 2000). Si la actividad (o parte del desarrollo de dicha actividad) ${ }^{2}$ se realiza con una instrucción “cara a cara”, el video proporciona al profesor la oportunidad de comprender e interactuar en mayor medida en relación al funcionamiento de su clase. El profesor puede observar muchos aspectos relacionados con la comunicación no verbal y lo que ocurre durante la actividad educativa (Heeler y Hardy, 2005) logrando una mejor atención a las demandas que se generan y por consiguiente, un mejor examen de las reacciones que se producen (Gamoran y Van Es, 2002).

Sin embargo, cabe destacar que no todas las películas de carácter documental son útiles para alcanzar aprendizaje (Poltorak, 1992). Un documental útil para emplear dentro del ámbito educativo debe presentar las siguientes características: 1) Proporcionar una organización jerárquica y una presentación del contenido; 2) Optimizar la experiencia educativa mediante la presentación de material a través de múltiples formas; 3) Proporcionar búsquedas flexibles mediante asociaciones de

\footnotetext{
2 Marquès (1999) distingue distintos tipos de videos educativos atendiendo a su estructura: a) documental, presenta ordenadamente información sobre un tema concreto, b) narrativo, tiene una trama narrativa a través de la cual va presentando la información, c) lección monoconceptual, vídeo de muy corta duración que presenta un único concepto, d) lección temática, el clásico vídeo didáctico que presenta un tema de manera sistemática y acorde a los destinatarios, e) vídeo motivador, pretende ante todo impactar, motivar, interesar aunque tenga que sacrificar la presentación sistemática del contenido y algo de rigor científico.
} 
datos; 4) Aumentar el contenido del video con material relevante procedente de fuentes externas (Dong y Li, 2005).

La literatura también ofrece un importante apoyo para la correcta utilización del material audiovisual a través de una serie de recomendaciones generales que permitirán al docente conseguir una mayor utilidad del empleo de material multimedia (Perry y Talley, 2001):1) El video general y el audio deben ser de excelente calidad; 2) El video debe incluir adicionalmente un soporte en texto; 3) El video en Internet no ha alcanzado todavía el grado de desarrollo suficiente para emplearlo en las aulas; 3) La actividad debe planificar un dialogo entre alumno y profesor; 4) Deben evitarse materiales de larga duración.

La consideración de todos estos factores que presenta la literatura debe contribuir a que se cumplan las funciones del vídeo con carácter educativo que, según Marqués (1999), pueden resumirse en las siguientes: proporcionar información que permita estructurar la realidad; instruir, orientar el aprendizaje y el desarrollo cognitivo; motivar, atraer, interesar y sensibilizar a los estudiantes; evaluar a través de la auto observación y el análisis; metalingüística, emplear el lenguaje audiovisual y expresivo a través de una técnica que permite modular y medir la expresividad de los estudiantes.

\subsection{La aplicación de películas documentales al estudio de casos empresariales}

La aplicación de la metodología del estudio de casos en la docencia de materias relacionadas con la empresa ha demostrado, durante décadas, ser un procedimiento en gran medida apropiado (Bonache, 1999; Gordon y Howell, 1959; Yacuzzi, 2005). Este técnica, aplicada a las organización y gestión de empresas, permite al alumno pensar y observar la resolución de situaciones y condicionantes en otras empresas, con las que puede encontrarse una vez finalizados sus estudios (Grávalos Gastaminza, 2001). Las experiencias empresariales que se conocen a través de casos facilitan, por tanto, el desarrollo integral del futuro profesional, completando el ciclo que forma el conocimiento generado y el conocimiento aplicado (Hennessey, 2003). 
Los estudios de casos de empresas en formato audiovisual, y particularmente de organizaciones interesantes y llamativas, han sido ampliamente analizados por la literatura como un medio que ha sido empleado de una manera satisfactoria en la enseñanza (Cannings y Talley, 2002; 2003; Perry y Talley, 2001; Sánchez et al., 2003). El número y relevancia de los artículos publicados con este argumento se fundamenta en que el estudio tradicional de casos puede extender su impacto a través de la utilización de las nuevas redes y herramientas de comunicación al alcance de los estudiantes tales como Internet y/o diversos dispositivos multimedia, entre los que cabe destacar el video (Cannings y Talley, 2002). Al presentar el caso al alumno a través de distintos medios y soportes, por ejemplo, complementando la información obtenida en el video con una búsqueda a través de Internet, se logra enriquecer la experiencia formativa lo que facilita la consolidación de aprendizajes y el desarrollo y la adquisición de conocimientos (Linderman et al., 1999).

Para el desarrollo efectivo del estudio de caso empresariales en video, Cannings y Talley, (2002) proponen que el docente tome en consideración los siguientes elementos: 1) Deben incluirse herramientas y recursos disponibles que permitan dialogo, una observación y una reflexión adicional. Estos recursos, que complementan la investigación, deben ser accesibles para el alumno. 2) El sistema de comunicación en curso debe permitir un dialogo en tiempo real del caso de estudio. 3) Para facilitar el visionado del caso se puede incluir pistas contextuales como textos, gráficos, audio, etc.

Los anteriores elementos permiten uno de los usos más comunes de los casos de estudio en video: "la reflexión en acción” (Valli, 1992). En muchas ocasiones se introducen las reflexiones del instructor o profesor en el estudio del caso a través de video, lo que ayudaría a los alumnos a desarrollar sus reflexiones y a participar en el dialogo. Este nuevo papel de los educadores requerirá un conjunto de habilidades diferentes que faciliten la observación directa e indirecta del alumnado, desarrollando nuevas formas de analizar las interacciones que tienen lugar en sus clases (Gamoran y Van Es, 2002). La observación puede llevarse a cabo a través de distintos materiales y fuentes que faciliten las reflexiones. El uso de casos en video facilita la puesta en común de la experiencia, guiando la discusión (Cannings y Talley, 2002; 2003). 


\section{Descripción de la experiencia}

\subsection{Contexto en el que surgió}

La enseñanza en el área de Organización de Empresas exige al docente acercar el mundo empresarial a los alumnos. Conscientes de esta necesidad, dos profesores del área decidimos aplicar una nueva herramienta a nuestras respectivas asignaturas, “Administración de Empresas y Organización de la Producción” de $3^{\circ}$ de Ingeniería Técnica Industrial e “Introducción a la Empresa” de $1^{\circ}$ de LADE.

Hasta el momento en que desarrollamos esta experiencia, nuestras actividades para lograr que los alumnos alcanzaran una visión holística (teórico - práctica) de la empresa, se basaban en el análisis de casos breves, obtenidos a través de bibliografía básica de la asignatura, y noticias procedentes de la prensa general y económica.

Sin embargo, tras recomendarnos el visionado de un documental, titulado "Planeta Zara” (coproducción de Produce + (Canal + España) y CapaLatAm) y un análisis en profundidad del mismo, decidimos utilizarlo como material docente para complementar las explicaciones teóricoprácticas. El documental se perfilaba como una herramienta muy útil y válida ya que retrataba la estructura, funcionamiento, estrategia y perspectivas de una de las mayores compañías textiles a nivel mundial. El hecho de utilizar un documental basado en el estudio de un caso de una empresa española atractiva y conocida por la totalidad del alumnado favorecía su interés en la actividad.

\subsection{Objetivos de la actividad}

En relación a los objetivos de la actividad se podían distinguir dos categorías de los mismos: generales y específicos:

\section{Objetivo general}

El principal objetivo de la experiencia era conseguir que los alumnos tuvieran un contacto directo con la realidad empresarial. Dada la dificultad de acceso a la información expuesta en el 
documental de una empresa de estas características, el empleo del esta herramienta debía permitir al alumnos acercarse a esta compañía.

\section{Objetivos específicos:}

En cuanto a objetivos más concretos y específicos se plantearon los siguientes:

1. Optimizar la actividad en el aula, al realizarse un trabajo previo a la proyección del documental. Se facilita de esta manera la labor de obtención de la información necesaria para el correcto desarrollo del trabajo posterior.

2. Mejorar los instrumentos utilizados para las aplicaciones prácticas que se realizan con los alumnos. El documental permite generar un entorno más innovador, con un mayor grado de motivación y concentración de los alumnos que en la clase presencial tradicional.

3. Favorecer el trabajo individual y en grupo. Un documental sobre una empresa conocida por todos los alumnos, resulta más atractivo para la participación activa a nivel individual y colectivo.

\subsection{Metodología}

La experiencia trataba de proporcionar una herramienta docente para el análisis conjunto e integrado de las diversas áreas de la empresa: Dirección Estratégica, RRHH, Marketing, Producción, Finanzas, Gobierno y Cultura Corporativa. Todas ellas son contempladas en los temarios de las asignaturas sujetas a estudio. El documental, como medio audiovisual, permite la aproximación directa del alumno al funcionamiento integral de la empresa y su entorno. Un instrumento que permite a los alumnos comprobar la aplicación práctica de la teoría, que en muchas ocasiones se pierde en el desarrollo de las “clases magistrales”.

El documental elegido fue "Planeta Zara” (2002), una coproducción de Produce + y CapaLatAm. La elección de este documental se debió a varios factores. En primer lugar, ofrece una perspectiva global del funcionamiento integral de una empresa conocida por todos los alumnos. En segundo lugar, posee una estructura dinámica y ágil que hace que el escenario del mismo se desplace en cortos períodos de tiempo a todos los puntos del planeta: Tokio, Nueva York, Milán, Paris, Emiratos Arabes Unidos, Arteixo, Tánger, transmitiendo la imagen de una firma internacional global. Además, el no excesivo metraje del documental, que tiene una duración aproximada de 55 
minutos, se adaptaba perfectamente al tiempo que se estimaba suficiente para la realización de la actividad y para completar todos los elementos previstos en el desarrollo de la experiencia.

Un aspecto clave a tener en cuenta para el desarrollo de la actividad es la definición clara del rol que debe desempeñar el profesor. En este sentido, se debe fomentar una actitud del docente que mejore y enriquezca el desarrollo de la actividad fomentando la participación de todos los alumnos y generando una correcta dinámica de grupo.

La actividad se llevó a acabo con dos grupos de asignaturas y estudios universitarios diferentes: Administración de Empresas y Organización de la Producción (último curso de la Ingeniería Técnica Industrial) e Introducción a la Empresa (primer curso de la Licenciatura de Administración y Dirección de Empresas). Ambas asignaturas tienen un carácter introductorio al estudio de la administración y organización de empresa. La experiencia se desarrollo durante una clase de 1 hora y 30 minutos de duración durante el primer cuatrimestre del curso académico 2006/07.

\subsection{Desarrollo}

La planificación de la actividad se llevó a cabo dividiendo la experiencia en dos partes. La primera se desarrolló en una clase de 1 hora y 30 minutos de duración. Se fraccionó la clase en tres periodos de tiempos diferenciados (Figura 1):

1. En los primeros 10 minutos los alumnos realizaron, de forma individual, un cuestionario previo a la proyección. Consideramos fundamental este trabajo inicial, y por ello, diseñamos un cuestionario que permitiese dirigir la atención de los alumnos a los aspectos del documental que cubrían los objetivos de aprendizaje perseguidos (Anexo 1). Posteriormente se puso en común en grupo las respuestas del cuestionario durante 5 minutos.

2. En una segunda parte, se proyecto el documental con una duración de 55 minutos. Los alumnos permanecieron muy atentos y tomando nota de los aspectos que se contemplaban en el cuestionario. 
3. La última parte de la práctica tuvo lugar en los 15 últimos minutos de la clase. Se puso en común los aspectos del documental recogidos en el cuestionario previo y aquellos que más habían llamado la atención de los alumnos. En esta parte es necesario que el profesor fomente un debate y participación dinámica de todos los miembros del grupo.

En la segunda parte de la experiencia se planteó una investigación en grupo sobre la empresa analizada en el documental. Para ello se proponía el uso de Internet como principal fuente de obtención de información. El objetivo de este trabajo era actualizar el documental con la situación actual de la empresa “Zara”. El documental, del año 2002, finalizaba con una planificación estratégica, a cuatro años vista, en la que se exponía las cifras de crecimiento a alcanzar en ese periodo. Al realizarse nuestra experiencia en el año 2006, se daban las circunstancias ideales para que los alumnos pudieran comprobar si, efectivamente, se habían alcanzado los objetivos planificados a largo plazo. Esta actividad pretendía que los alumnos realizasen búsquedas a través de Internet y accedieran a información primaria y secundaria de la empresa, así como a datos económico - financieros de la compañía y analizasen el proceso de crecimiento de la empresa. Para la realización de esta segunda fase del trabajo se dio un plazo de una semana, cumplido dicho plazo los resultados se entregarían bien personalmente en la clase, a través del correo electrónico del profesor o a través de Internet utilizando el campus virtual con el que cuenta la Universidad Rey Juan Carlos.

Para finalizar con la experiencia, y una vez terminada la actividad se distribuyeron cuestionarios de evaluación entre los estudiantes. El objetivo de los mismos era tratar de conocer de forma directa las opiniones, comentarios y valoraciones de los alumnos en relación con la actividad desarrollada así como conseguir información que permitiese mejorar el desarrollo y realización de la actividad en sucesivos cursos académicos. 


\subsection{Resultado de la actividad}

Para la valoración de la actividad se empleó como técnica de recogida de información un cuestionario que se facilitó una vez completada la aplicación práctica. El cuestionario planteaba que los alumnos valorasen tanto el contenido de la actividad como la organización de la misma. Para ello, se empleó una escala de Likert de cinco puntos, en la cual “uno” representaba el máximo desacuerdo y “cinco” el máximo acuerdo con la afirmación planteada en el cuestionario.

A partir de los resultados obtenidos una primera valoración a nivel general podría afirmarse que la experiencia ha resultado muy satisfactoria para los alumnos. Este resultado coincide en los dos grupos dónde se desarrolló la experiencia, no habiéndose encontrado diferencias significativas entre los mismos a excepción de algún aspecto que se comentará a continuación lo que hace que la evaluación de los resultados se haga sobre el total de alumnos que han participado en la misma sin discriminar el grupo en el que estuvieran matriculados.

En cuanto a la valoración del contenido de la actividad, todos los aspectos a considerar obtuvieron una alta puntuación, tal y como puede comprobarse en la Tabla 1 y la Figura 2. Cabe destacar que el punto en que mayor diversidad se obtuvo, entre los resultados de los alumnos de las dos asignaturas, se encontró en la valoración del tiempo disponible para el trabajo posterior al documental. Se obtuvo una menor valoración en los alumnos de la Ingeniería Técnica Industrial, motivado, quizás, por la mayor carga de trabajos prácticos y laboratorios a realizar fuera del horario lectivo derivados de la dinámica habitual de los estudios de ingeniería.

De los resultados obtenidos en relación al contenido de la actividad, los alumnos han valorado de forma muy positiva tanto la actualidad e interés del tema presentado (a.1) como la relevancia de la empresa de la actividad. De hecho, todos los alumnos afirmaban haber entrado en alguna ocasión en un establecimiento del Grupo Inditex lo que confirma la expansión que ha alcanzado esta compañía dentro del territorio nacional y el interés y admiración que despierta entre los alumnos.

En relación a los tiempos de desarrollo de los distintos trabajos que conformaban la actividad, los alumnos se muestran conformes con la duración del documental (55 minutos) pero 
muestran valoraciones inferiores tanto en lo que se refiere al tiempo disponible para la actividad en grupo previa (a.4) como para la realización del trabajo posterior al documental (a.6) lo que puede indicar la necesidad de conceder más tiempo para la realización de estos aspectos.

Por último, la elevada valoración de la aplicación práctica del documental parece confirmar que el material audiovisual consiguió uno de los objetivos propuestos al plantear la actividad que era el acercamiento de la realidad empresarial a los alumnos, configurándose este material como una herramienta válida para la consecución de este objetivo

Tabla 1: Valoración del contenido de la actividad por los alumnos

\begin{tabular}{|c|c|c|c|c|c|c|c|}
\hline VALORACIÓN DEL CONTEN & $\mathbf{1}$ & 2 & 3 & 4 & 5 & MODA & MEDI A \\
\hline a.1. Actualidad e interés de los temas presentados & 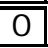 & $\overline{11}$ & 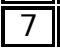 & 27 & 27 & 4 & 4,29508 \\
\hline a.2. Aplicabilidad práctica & 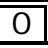 & 1 & 11 & 39 & 11 & 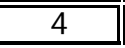 & 3,96721 \\
\hline a.3. Tiempo disponible para el cuestionario individual previo & 0 & 3 & 18 & 24 & 17 & 4 & 3,88525 \\
\hline a.4. Tiempo disponible para la actividad en grupo previa & 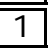 & 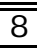 & 19 & 22 & 7 & 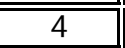 & 3,44643 \\
\hline a.5. Tiempo duración documental & $\overline{0}$ & $\overline{4}$ & 16 & 32 & 10 & $\overline{4}$ & 3,77049 \\
\hline a.6. Tiempo disponible para el trabajo posterior al & 1 & 5 & 26 & 23 & 4 & 3 & 3,41379 \\
\hline a.7. Relevancia de la empresa de la actividad & 0 & $\overline{0}$ & 6 & 19 & 37 & $\overline{5}$ & 4,5 \\
\hline
\end{tabular}

Figura 2: Media de las valoraciones del contenido de la actividad

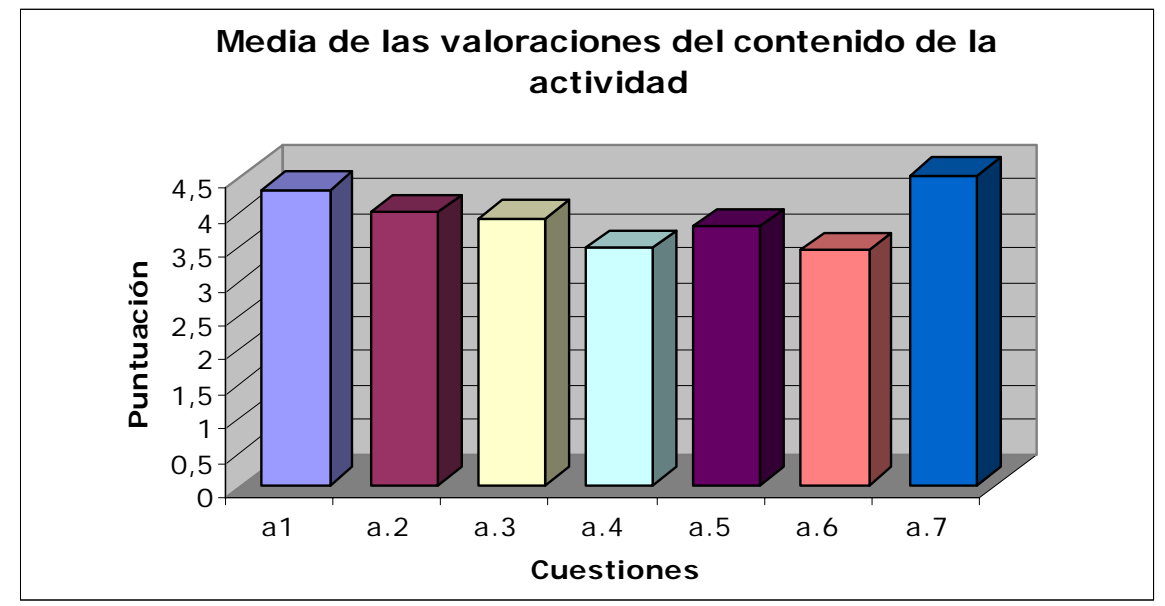

En relación a la valoración de la organización de la actividad (Tabla 2 y Figura 3), uno de los aspectos más valorados en la organización de la actividad fue la realización de un trabajo previo a la proyección para su mejor comprensión y análisis. Los alumnos parecen reconocer que, de esta manera, se consigue facilitar el análisis del material audiovisual y centrar a los alumnos en aquellos 
aspectos en los cuáles se quería poner más énfasis, tomando como punto de partida la gran cantidad de información que proporciona.

Aunque la organización general de la actividad (b.7) ha sido el aspecto más valorado por los alumnos por los alumnos, debe puntualizarse la importancia de la realización de una prueba tanto de audio como de visión en el aula donde se realizará la proyección. La falta de esta prueba no permitió comprobar ciertos problemas de audición en la primera emisión, que fueron subsanados en la segunda. Los aspectos técnicos de la actividad deben ser comprobados de forma previa, tanto en audición como en visionado, esto evitará pérdidas de tiempo en el desarrollo de la jornada de proyección, ya que romperá el ritmo de trabajo establecido.

Las cuestiones que obtienen valoraciones más bajas vuelven a hacer referencia a la concesión de tiempos para la realización de ciertas actividades (b.4), así como al momento del curso académico en que se desarrolló la actividad (b.5). En relación a estos aspectos cabe destacar que se considera adecuada la realización de la actividad en aproximadamente 2 horas y media de clase y que, por otro lado, debe elegirse como momento para la proyección de la actividad aquel en el cual los alumnos disponen de los conocimientos teóricos necesarios como para ser capaces de asimilar los objetivos fundamentales definidos para la actividad.

Tabla 2: Valoración de la organización de la actividad

\begin{tabular}{|c|c|c|c|c|c|c|c|}
\hline VALORACIÓN DE & 1 & 2 & 3 & 4 & 5 & MODA & MEDI A \\
\hline b.1. Publicidad del Seminario adecuada & $\overline{0}$ & 1 & 111 & 18 & 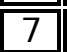 & 4 & $\overline{3,83333}$ \\
\hline b.2. Proceso sencillo de entrega de los trabajos & $\overline{0}$ & 0 & 15 & 31 & 16 & 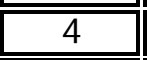 & 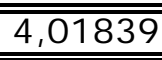 \\
\hline $\begin{array}{l}\text { b.3. ¿Te ha parecido adecuado que la actividad se realizara en una } \\
\text { tarde? }\end{array}$ & 0 & 3 & 11 & 29 & 19 & 4 & 4,03279 \\
\hline b.4. Número de horas por tarde adecuado & 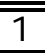 & 10 & 12 & $\mid 28$ & 111 & 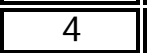 & 3,60656 \\
\hline $\begin{array}{l}\text { b.5. Momento del curso académico en que se ha organizado el } \\
\text { Seminario adecuado }\end{array}$ & 0 & 1 & 4 & 31 & 24 & 4 & 3,77966 \\
\hline $\begin{array}{l}\text { b.6. Valora la realización de un trabajo previo a la proyección del } \\
\text { documental para su mejor comprensión y análisis }\end{array}$ & 3 & 6 & 17 & 27 & 9 & 4 & 4,03279 \\
\hline b.7. Valora la organización de la actividad a nivel general & $\overline{0}$ & $\overline{0}$ & 3 & 39 & 20 & 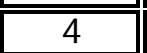 & 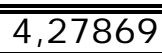 \\
\hline
\end{tabular}

Figura 3: Medida de las valoraciones de la organización de la actividad alumnos 


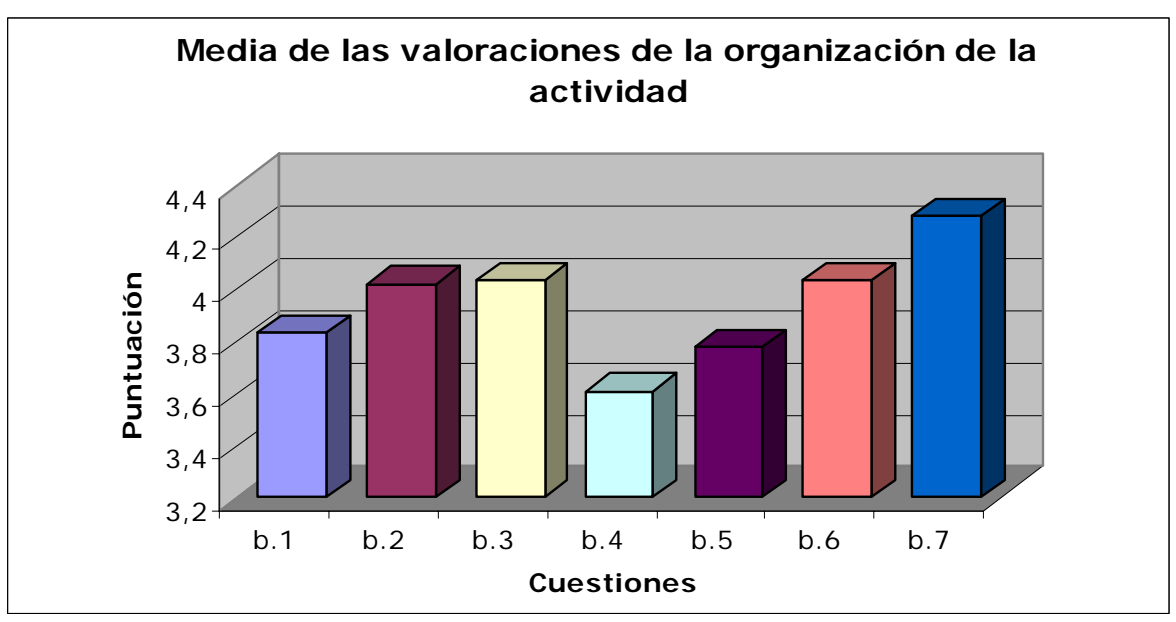

\section{Conclusiones}

En el marco del Espacio Europeo de Educación Superior (EEES) la docencia universitaria se encamina hacia un nuevo modelo de enseñanza. En este modelo pierde vigencia la figura del profesor como mero transmisor de conocimientos a través de la lección magistral que, posteriormente, son evaluados en una prueba escrita. En el nuevo contexto, el alumno adquiere un papel más activo y el profesor debe adaptarse a esa nueva forma de enseñar. Las clases presenciales pierden peso y las tutorías y la realización de actividades relacionadas con las materias impartidas a los alumnos ganan peso de manera significativa.

Es en este marco donde la búsqueda y utilización de nuevas técnicas y metodologías docentes que ayuden a dinamizar y mejorar la transmisión de conocimientos a los alumnos adquieren todo su sentido. En particular, este trabajo describe la experiencia docente de dos profesores del área de Organización de Empresas que tienen como objetivo formar a sus alumnos en aspectos relacionados con la organización, gestión, dirección y estrategia de la empresa.

El empleo de materiales audiovisuales y en particular de películas de carácter documental que describen experiencias de empresas y organizaciones de éxito y singulares en su proceso de crecimiento, desarrollo o estrategia han sido considerado como herramientas útiles para la docencia tanto a nivel de estudiantes universitarios como de directivos. 
A partir de la experiencia desarrollada y a la vista de los resultados obtenidos, derivados de las valoraciones realizadas por los estudiantes, se puede concluir que la utilización de material audiovisual ha proporcionado a los alumnos una experiencia positiva. En primer lugar, porque la proyección de este documental les ha permitido obtener un mayor nivel de conocimiento del mundo empresarial y en particular de una empresa que todos ellos conocen y con la que se han relacionado en alguna ocasión. En segundo lugar, porque se han alcanzado mayores niveles de motivación y satisfacción de la experiencia que los derivados de la tradicional lección magistral y, por último, porque el empleo de diversas fuentes de información ha contribuido a mejorar y consolidar el aprendizaje de los alumnos y a investigar aspectos de la empresa que hubieran sido complicados con el formato tradicional de las clases a través de la lección magistral.

A pesar de la que la experiencia desarrollada ha alcanzado únicamente a dos grupos la satisfacción tanto de los docentes como de los alumnos nos lleva a recomendar el empleo de esta técnica en diversas áreas docentes universitarias y a compartir y generar experiencias que conduzcan a los docentes a disponer de más y mejores técnicas para la transmisión de conocimientos a los estudiantes. 


\section{Anexo I}

11 de Diciembre de 2006; tiempo para su realización: $10 \mathrm{~min}$.

Apellidos y Nombre

Grupo

CUESTIONARIO PREVIO DOCUMENTAL

"PLANETA ZARA"

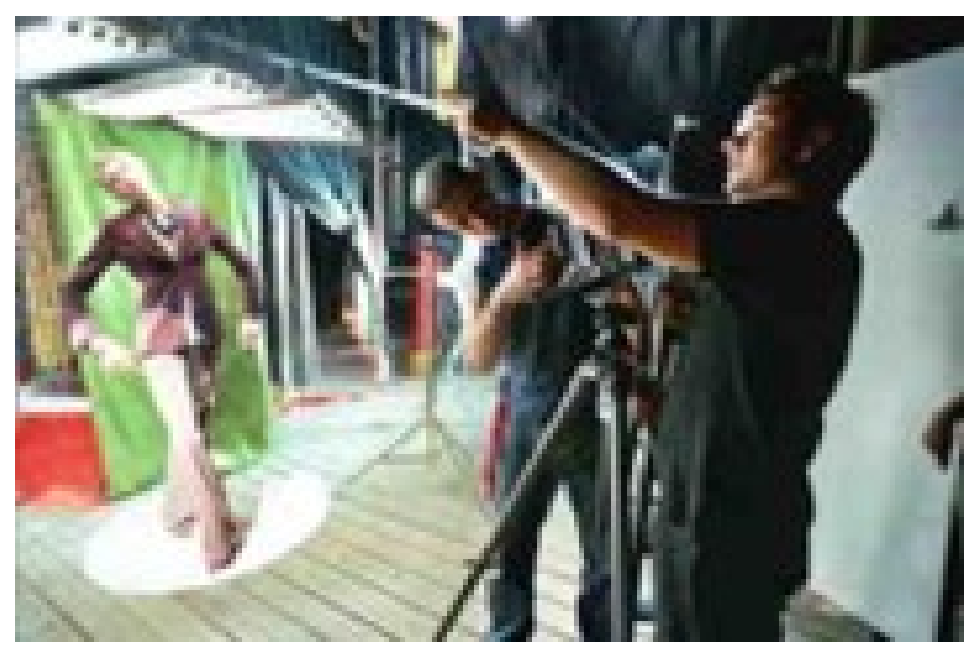


Antes de la proyección del documental "Planeta Zara”, complete el siguiente cuestionario señalando la opción que considere correcta.

1. El grado de diferenciación de Zara en relación a sus competidores se logra a través de:

a. Una moda exclusiva y de mayor calidad que la de los competidores.

b. Una moda fugaz y asequible que se vende fácilmente.

c. Ofrece una moda muy similar a la de sus competidores con un escaso nivel de diferenciación.

2. La segunda dimensión de la que depende la diferenciación es el grado de eficiencia relativa (los costes). Para ello la producción de Zara:

a. Se deslocaliza completamente a países del Tercer Mundo (Marruecos, Asiáticos, etc).

b. El $80 \%$ de la producción es nacional lo que le permite rapidez de reacción y sólo la producción de las prendas más elaboradas se deslocaliza.

c. La producción es totalmente nacional ya que de esta manera se permite un mayor control y aprovechamiento de la materia prima.

3. Respecto a la estrategia corporativa de Zara relacionadas con el desarrollo de la producción y almacenaje, se puede afirmar que:

a. Las tiendas cuentan con grandes almacenes que les permiten un abastecimiento rápido para que el cliente encuentre variedad de tallas y modelos.

b. Existe un centro de distribución conectado con las fábricas y que permiten que las tiendas no necesiten almacén y puedan dedicar toda su superficie a la venta.

c. El centro de distribución y los almacenes de las tiendas son reducidos para poder hacer frente a los rápidos cambios en la demanda y gusto de los consumidores

4. El proceso de internacionalización de Zara se inició a finales de los 80 . ¿Qué ritmo de apertura considera que ha alcanzado la empresa?:

a. Una tienda cada mes.

b. Una tienda a la semana.

c. Una tienda cada seis meses.

5. Cuál de las siguientes opciones refleja mejor la manera en la que Zara desarrolla su publicidad:

a. A través de importantes campañas de publicidad en distintos medios de comunicación.

b. Zara no hace publicidad. La imagen de la marca la dan los escaparates y la ubicación de las tiendas es fundamental.

c. Con modelos conocidas que representan la imagen de Zara en catálogos y anuncios. 
6. Respecto a las características de la política de personal se podría destacar:

a. La gran autonomía de las jefas de tienda que les permite elegir la ropa que se venderá en sus tiendas y tener línea directa con Amancio Ortega.

b. Un gran control por parte de la central de Arteixo de las responsables de las tiendas marcándoles la cantidad y variedad de artículos que se sirve a su tienda.

c. Se le concede una mayor autonomía a aquellas jefas de tienda con mayor permanencia en la empresa y con mayor edad, manteniendo con ellas una comunicación más abierta.

7. El diseño de las nuevas prendas que se producirán dependerá:

a. En exclusiva de los diseñadores que captan en la calle las nuevas tendencias que el cliente marca.

b. El diseñador somete a la aprobación de los comerciales cada nueva prenda para que sea producida.

c. Los comerciales aportan ideas a los diseñadores, pero son estos últimos los que tienen el poder de decisión sobre qué diseñar.

8. Amancio Ortega representa a un tipo de empresario con las siguientes características:

a. Es accesible a los medios de comunicación y los utiliza para un mayor conocimiento de la marca a nivel internacional.

b. No concede entrevistas $y$ no asiste a las inauguraciones de sus tiendas. Aunque mantiene un contacto personal y continuo con sus empleados.

c. Debido a la complejidad y tamaño que ha alcanzado Zara mantiene contacto con los directivos intermedios que funcionan como canal de comunicación.

9. Desde su salida en Bolsa, los grandes grupos de inversión (los nuevos dueños de la empresa) tienen como objetivo principal:

a. El crecimiento continuado de los beneficios en la cotización bursátil.

b. El control de la toma de decisiones en distintas áreas como la producción, expansión de la empresa o dirección de personal.

c. Conocer dónde la empresa va a estar en el futuro: crecimiento, número de tiendas, mercados, etc.

10. Otra de las preocupaciones que tuvo que afrontar en su salida a Bolsa fue su relación con los países pobres, que afrontó:

a. Desarrollando una globalización ética cuya labor se facilita a través de la colaboración con ONGs.

b. Permitiendo la subcontratación de su producción lo que le eximía legalmente de responsabilidad y de las revisiones de los talleres colaboradores. Esto ha supuesto una gran reducción en los costes de producción.

c. Reduciendo la producción en países subdesarrollados o trasladando la producción a aquellos cuya legislación es más flexible. 


\section{Bibliografía}

- AGUILERA, M.; MÉNDIZ, A. (2003): "Video games and education (Education in the face of a “parallel school”)”. ACM Computers in Entertainment, vol. 1, n. 1, pp. 1-14.

- BACHMAIR, B. (1996): Fernsehkultur - Subjektivität in einer Welt bewegter Bilder. Opladen: Westdeut-scher Verlag, pp. 339-357

- BONACHE, J. (1999): “El estudio de casos como estrategia de construcción teórica: características, críticas y defensas”. Cuadernos de Economía y Dirección de la Empresa, n. 3, Ene-Juni, pp. 123-140.

- CANNINGS, T.; TALLEY, S. (2002): “Multimedia and online video case studies for preservice teacher preparation”. Education and Information Technologies, vol. 7, n. 4, pp 359-367.

- CANNINGS, T.; TALLEY, S. (2003): "Bridging the gap between theory and practice in preservice education: The use of video case studies”. Proceedings of the 3.1 and 3.3 working groups conference on International federation for information processing: ICT and the teacher of the future, vol. 23. The University of Melbourne, Australia.

- DONG, A.; HONGLIN, L. (2005): “Educational documentary video segmentation and access through combination of visual, audio and text understanding”. IEEE International Symposium on Signal Processing and Information Technology, pp. 652-657.

- EARLEY, P. CH. (1987): "Intercultural training for managers: A comparison of documentary and interpersonal methods”. The Academy of Management Journal, vol. 30, n. 4, pp. 685-698.

- GAROMAN, M.; VAN ES, E. (2002): “Using video to support teachers" ability to interpret classroom: interactions”. Technology and teacher education annual, vol. 4, pp. 2532-2536.

- GODMILOW, J.; SHAPIRO, A. (1997): “How real is the reality in documentary film?”. History and Theory, vol. 36, n. 4.

- GÖTZ, M. (2005): “Learning in knowledge and documentaryprogrammes”. Journal Televizione, n. 18, pp. 24-33.

- GRÁVALOS, M. A. (2001): “Métodos de enseñanza en organización y gestión de empresas turísticas”. Trabajos Investigación Universitaria Turísticas. ANESTUR

- HEELER, PH.; HARDY, C. (2005): “A preliminary report on the use of video technology in online courses”. Journal of Computing Sciences in Colleges, vol. 20, n. 4, pp.127-133.

- HENNESSEY, G. (2003): “El proceso enseñanza-aprendizaje de la comunicación organizacional. Un proceso de formación de consultores basado en un modelo de una acciónreflexión empresa-aula y el aprendizaje autónomo”. Razón y palabra, n. 32. 
- KOLB, D.; FRY, R. (1975): “Toward an applied theory of experimental learning”. In C.L. Cooper (Ed.). Theories of group processes (pp. 33-58). New York: John Wiley and Sons.

- LIENHART, R.; PFEIFFER, S.; EFFELSBERG, W. (1997): “Video Abstracting”. Communication of the ACM, vol. 40, n. 12, pp. 55-63.

- MARQUÉS, P. (1999): “Los videos educativos: Tipología, funciones y orientaciones para su uso”. Enciclopedia Virtual de Tecnología Educativa. http//dewey.uab.es

- MERCEY, A. A. (1939): “Teaching social studies through documentary films”. The Journal of Higher Education, vol. 10, n. 6, pp. 303-308.

- MIKOS, L. (2001): “Fern-Sehen”. Bausteine zu einer Rezaptionsästhetik des Fernsehens. Berlín.

- MOFFATT, M. P.; RICH, S. G. (1951): “What documents have educational value?”. Journal of Educational Sociology, vol. 25, n. 1, pp. 23-33.

- MOSS, L. S. (1979): “Film and the transmission of economic knowledge: A report”. Journal of Economic Literature, vol. 17, n. 3, pp. 1005-1019.

- PERRY G.; TALLEY S. (2001): “Online video case studies and teacher education: A new tool for pre-service teacher education”. Journal of Computing in Teacher Education, vol. 17, n.4, pp.6-31.

- PHILIPS, H. W. (2002): “Alternative to live films: Documentary films in action”. Film an introduction (pp. 101). New York: Bedfat / St. Martins.

- POLTORAK, D. (1992): "Problems of perception of audio-visual information in studying History”. The History Teacher, vol. 25, n. 3, pp. 313-319.

- $\quad$ PUTNAM, R. T.; BORKO, H. (2000): "What do new views of knowledge and thinking have to say about research on teacher learning?”. Educational Researcher, vol. 29, n.1 pp. 4-15.

- $\quad$ RAACK, R. C. (1972): “Clio's dark mirror: The documentary film in History”. The History Teacher, vol. 6, n. 1, pp. 109-118.

- $\quad$ RAACK, R. C.; SMITH, A. M.; RAACK, M. L. (1973): “The documentary film in History teaching: An experimental course”. The History Teacher, vol. 6, n. 2, pp. 281-294.

- $\quad$ ROGERS, Y.; SCAIFE, M. (1998): “How can interactive multimedia facilitate learning?”. In Lee. J., (Ed.). Intelligence and Multimodality in Multimedia Interfaces: Research and Application, AAAI. Press: Menlo Park, CA.

- SÁNCHEZ, J. A.; BRAVO, J. L.; FARJAS, M.; VÁZQUEZ, A. (2003): “Innovaciones didácticas en aulaweb: El estudio de casos en la titulación de ingeniería técnica topográfica”. Congreso Nuevas Tecnologías en la Innovación Educativa, pp. 209-217. 
- SMITH, T.; RUOCCO, A.; JANSEN, B. (1999): “Digital video in education”. The 30th ACM SIGCSE Technical Symposium, New Orleans, LA.

- $\quad$ SPRAGUE, D.; KOPFMAN, K.; DE LEVANTE, D. S. (1998): "Faculty development in the integration of technology in teacher education courses”. Journal of Computing in Teacher Education, vol. 14, n. 2, pp. 24-28.

- VALLI, L. (1992): “Reflexive teacher education”. Albano, New York, State University of New York.

- WordNet, http:// www.cogsci.princeton.edu/cgi-bin/webwn2.1

- YACUZZI, E. (2005):“El estudio de caso como metodología de investigación: Teoría, mecanismos causales, validación”. CEMA Working Papers. Universidad de CEMA. 